\title{
Conditions for the loss of stability of eqiulibrium manifold in satellite model
}

\author{
E. Shchepakina ${ }^{1}$, V. Sobolev ${ }^{1}$ \\ ${ }^{1}$ Samara National Research University, 34 Moskovskoe Shosse, 443086, Samara, Russia
}

\begin{abstract}
The problem of stabilizing a spin satellite by means of passive dampers is considered. The application of the method of integral manifolds allows us to find conditions for the loss of stability in the analytical form.
\end{abstract}

Keywords: stability; stabilization; manifold of steady states; satellite

\section{Introduction}

A lot of work has been devoted to the study of dynamic models of stabilization of satellites with the help of gyroscopic forces. As the main apparatus, the Lyapunov function method and the stability criteria applied to first approximation systems are used. In addition to gyroscopic forces for stabilization, damping devices are used in a number of models to ensure the asymptotic stability of the required modes of satellite motion. In a number of works, passive dampers are considered as such devices. For the case of two co-axial bodies, on each of which one damper is installed, the stabilization problem was considered, for example, in [1-3]. In this paper, we confine ourselves to the study of a model of a satellite consisting of two bodies, on one of which a damper with a relatively small coefficient of viscous friction is installed. The damper is modeled by a particle of relatively small mass placed in a tube filled with a viscous liquid and attached by a spring. To analyze the system of differential equations, the method of integral manifolds [3, 4] is applied, which allows to significantly reduce the dimensionality of the model and simplify the analysis.

\section{Equations}

To study the conditions and the mechanism of loss of stability for a satellite stabilized by rotation, consider a dynamic model that is a system of ordinary differential equations for dimensionless variables and parameters of the form [3]:

$$
\begin{gathered}
q \dot{\omega}-\varepsilon \dot{x_{1}}=\varepsilon\left[2 x_{1} v_{1}-\omega x_{2} u_{1}\right], \\
{\left[1-2 L u_{1}\right] \dot{x_{1}}-\varepsilon u_{2} \dot{\omega}=} \\
=-\Lambda x_{2}+\varepsilon\left[-u_{1} 2 L \omega x_{2}+\varepsilon x_{1} x_{2} u_{1}+2 L x_{1} v_{1}\right], \\
{\left[1-2 L u_{1}\right] \dot{x_{2}}-\varepsilon \dot{v}_{1}=\Lambda x_{1}+\varepsilon\left[\omega^{2} u_{1}-2 L u_{1} \omega x_{1}+2 L x_{2} v_{1}-\varepsilon x_{1}^{2} u_{1}\right]} \\
\dot{u}_{1}=v_{1}, \\
-\varepsilon \dot{x_{2}}+\varepsilon\left(1-\varepsilon \rho_{1}\right) \dot{v_{1}}= \\
=-K_{1} u_{1}-\varepsilon \beta_{1} v_{1}+\varepsilon\left(x_{1}^{2}+x_{2}^{2}\right)\left(u_{1}-L\right)-\varepsilon \omega x_{1} .
\end{gathered}
$$

Variables $\omega, x_{1}, x_{2}$ play the role of projections of the absolute angular velocity of the main body on the axis of the coordinate system associated with it with the origin at the center of mass of this body. The variable $u_{1}$ characterizes the deviation of a particle moving inside the damper from its nominal position. In these equations, the nonlinear terms containing the factors $\varepsilon^{2} u_{1}$ are omitted. The value of $\varepsilon$, which characterizes the moment of inertia of the mass moving in the damper, plays the role of a small parameter. Some details can be found in [5].

\section{Manifold of steady states}

The system of differential equations under consideration has a manifold of steady states:

Following [6], we say that this manifold is stable with respect to variables

$$
\mathfrak{M}=\left\{\omega=\Omega=\text { const }, \quad x_{1}=x_{2}=u_{1}=v_{1}=0\right\} .
$$

$$
x_{1}, x_{2}, u_{1}, v_{1}
$$

If for any $\omega=\Omega$ and any neighborhood of zero $W$ in the space of variables $x_{1}, x_{2}, u_{1}, v_{1}$ we can find a neighborhood of zero $W_{0}$ of this space such that for any point of this neighborhood the corresponding solution belongs to $W$ for $t \geq 0$.

We will say that $\mathfrak{M}$ is asymptotically stable with respect to variables

$$
x_{1}, x_{2}, u_{1}, v_{1} \text {, }
$$

if it is stable with respect to these variables and, in addition, the variables $x_{1}, x_{2}, u_{1}, v_{1}$ tend to zero with unlimited increase of $t$. 
We will say that $\mathfrak{M}$ is stabilizable if it is asymptotically stable with respect to variables $x_{1}, x_{2}, u_{1}, v_{1}$ under $t \rightarrow \infty$ the solution tends to some point of the manifold $\mathfrak{M}$.

It follows from the results of $[5,6]$ that the manifold of steady states $\mathfrak{M}$ is stabilizable if all the roots of the characteristic equation, except for one zero root, have negative real parts. Any perturbed motion, sufficiently close to the unperturbed motion, tends to one of the possible steady motions belonging to the indicated manifold if $t \rightarrow \infty$.

\section{Model reduction}

The differential system under consideration is singularly perturbed one and has a three-dimensional manifold of slow motions:

$$
u_{1}=\varepsilon f\left(\omega, x_{1}, x_{2}\right), \quad v_{1}=\varepsilon g\left(\omega, x_{1}, x_{2}\right),
$$

the motion along which is described by a system of three scalar differential equations of the form:

$$
\begin{aligned}
& q \dot{\omega}=\varepsilon\left[2 x_{1} g-(\Lambda+\omega) x_{2} f\right] \text {, } \\
& \dot{x_{1}}=-\Lambda x_{2}+\varepsilon\left[x_{2}\left(x_{2}-2 L \omega(\Lambda+\omega)\right)+f+2 L x_{1} g\right], \\
& \dot{x_{2}}=\Lambda x_{1}+\varepsilon\left[\left(-K_{1} f-x_{1}\left(x_{1}-2 L \omega(\Lambda+\omega) x_{2}\right) f+x_{1}^{2}+x_{2}^{2}-\left(1+\rho_{1}\right) K_{1}+\omega^{2}\right) f+\right. \\
& \left.\left(-\beta_{1}+2 L x_{2}\right) g+(\Lambda-\omega) x_{1}-L\left(x_{1}^{2}+x_{2}^{2}\right)\right]+ \\
& \varepsilon^{2}\left\{\left[\omega^{2}-\left(1+\rho_{1}\right)^{2} K_{1}\right] f-\left(1+\rho_{1}\right) \beta_{1} g+\left(1+\rho_{1}\right) \omega x_{1}-\left(1+\rho_{1}\right)\left(x_{1}^{2}+x_{2}^{2}\right)\right\}+\varepsilon^{3}\left(1+\rho_{1}\right)^{2}(\Lambda-\omega) x_{1} .
\end{aligned}
$$

The functions $\mathrm{f}, \mathrm{g}$ are computed in the usual way [5]. Restricting ourselves linearly in $x_{1}, x_{2}$ terms to the third order and nonlinear - up to the second order in $\varepsilon$ inclusive, we write the equations of motion with respect to the integral manifold in the form

$$
\begin{aligned}
& q \dot{\omega}=\frac{\varepsilon^{2}}{K_{1}}\left[-(\Lambda-\omega)(3 \Lambda+\omega) x_{2} x_{1}+(\Lambda+\omega) L x_{2}\left(x_{1}^{2}+x_{2}^{2}\right)\right] \\
& \dot{x_{1}}=-\Lambda x_{2}+\frac{\varepsilon^{2}}{K_{1}}\left[(\Lambda-\omega) x_{1}^{2} x_{2}-2 L(\Lambda-\omega)(2 \Lambda+\omega) x_{2} x_{1}+\right. \\
& \left.2 L^{2}(\Lambda+\omega) x_{2}\left(x_{1}^{2}+x_{2}^{2}\right)-L x_{1} x_{2} x_{1}\left(x_{1}^{2}+x_{2}^{2}\right)\right] \\
& \dot{x_{2}}=\Lambda x_{1}+\varepsilon^{2}\left[-\frac{1}{K_{1}}(\Lambda+\omega)(\Lambda-\omega)^{2}\left(1-\frac{\varepsilon L^{2}}{K_{1}}\right) x_{1}-\frac{\varepsilon}{K_{1}^{2}}\left(\Lambda(\Lambda+\omega)(\Lambda-\omega)^{2} x_{2} \beta_{1}\right)+\frac{1}{K_{1}} 2 L(\Lambda-\omega)\left((\Lambda+\omega) x_{1}^{2}-\Lambda x_{2}^{2}\right)\right. \\
& \left.-2 L(\Lambda+\omega) x_{1}\left(x_{1}^{2}+x_{2}^{2}\right)+L\left(x_{1}^{2}-\omega^{2}\right)\left(x_{1}^{2}+x_{2}^{2}\right)\right] .
\end{aligned}
$$

After linearizing the equations on an integral manifold for variables $x_{1}, x_{2}$ we obtain the linear with respect to $x_{1}, x_{2}$ subsystem

$$
\begin{gathered}
\dot{x_{1}}=-\Lambda x_{2}, \\
\dot{x_{2}}=\Lambda x_{1}+\varepsilon^{2}\left[-\frac{1}{K_{1}}(\Lambda+\omega)(\Lambda-\omega)^{2}\left(1-\frac{\varepsilon L^{2}}{K_{1}}\right) x_{1}-\frac{\varepsilon}{K_{1}^{2}}\left(\Lambda(\Lambda+\omega)(\Lambda-\omega)^{2} x_{2} \beta_{1}\right)\right] .
\end{gathered}
$$

The condition of asymptotic stability with respect to variables $x_{1}, x_{2}$ is

$$
-\Lambda(\Lambda+\omega)(\Lambda-\omega)^{2}<0
$$

For the integral manifold of slow motions, the following principle is valid: the variety of stationary states of the initial system is stable (unstable, asymptotically stable with respect to some of the variables, is stabilizable) if and only if it is stable (unstable, asymptotically stable with respect to a part of the variables, stabilizable) the variety of stationary states of a system describing the motion on an integral manifold. It is clear that a violation of the resulting inequality entails a loss of stability. This is confirmed by the results of numerical experiments. In the figures below, one can see oscillations with increasing amplitude for the variables $x_{1}, x_{2}$ and $\omega$.

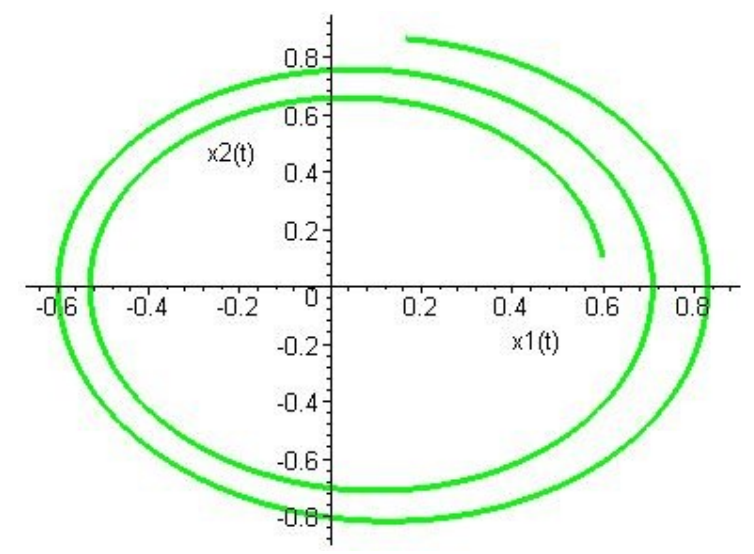

Fig. 1. Projection of the trajectory on the plane of variables $x_{1}, x_{2}$ (the movement is made counter-clockwise). 




Fig 2. Solution graph for variable $\omega$.

\section{Conclusion}

In the present work, the mathematical model of a satellite stabilized by rotation has been studied by the methods of the geometric theory of singular perturbations. A reduction of the system was carried out, as a result of which, instead of the original system of five differential equations, its projection onto a three-dimensional slow integral manifold was investigated. It should be noted that, due to the validity of the reduction principle for a slow integral manifold, the reduction is carried out correctly, and the reduced system of three differential equations preserves the basic qualitative properties of the original model. An inequality is obtained, in violation of which the satellite loses the required orientation in space.

\section{Acknowledgements}

The study was carried out with the financial support of the RFBR and the Government of the Samara Region within the framework of the scientific project No. 16-41-630524 and the Ministry of Education and Science of the Russian Federation as part of the Samara University's competitiveness increase program (2013-2020).

\section{References}

[1] Teixeira-Filho DR, Kane DR. Spin stability of torque free systems. Part I, II. AIAA Journal 1973; 11(6): 862-867.

[2] Mingori D. Effect of energy dissipation on the attitude stability of dualspin satellites. AIAA Journal 1969; 7(7): 862-867.

[3] Strygin VV, Sobolev VA. Effect of geometric and kinetic parameters and energy dissipation on orientation stability of satellites with double spin. Cosmic Research 1976; 14: 331-335.

[4] Shchepakina E, Sobolev V, Mortell MP. Singular Perturbations. Introduction to system order reduction methods with applications, 2014 ; 121 p.

[5] Strygin BB, Sobolev VA. Decomposition of motions by the Integral Manifolds Method. Moscow: Nauka, 1988. (in Russian)

[6] Aizerman MA, Gantmakher FR. Stabilität der Gleichgewichtslage im einem night-holonomen System. Z. angew. Math, und Mcch. 1957; 37(1/2): 74-75. 